\title{
Preparing children with a mock scanner training protocol results in high quality structural and functional MRI scans
}

\author{
Henrica M. A. de Bie • Maria Boersma • Mike P. Wattjes • Sofie Adriaanse • \\ R. Jeroen Vermeulen • Kim J. Oostrom • Jaap Huisman • Dick J. Veltman • \\ Henriette A. Delemarre-Van de Waal
}

Received: 18 January 2010 / Accepted: 23 February 2010 /Published online: 13 March 2010

(C) The Author(s) 2010. This article is published with open access at Springerlink.com

\begin{abstract}
We evaluated the use of a mock scanner training protocol as an alternative for sedation and for preparing young children for (functional) magnetic resonance imaging (MRI). Children with severe mental retardation or developmental disorders were excluded. A group of 90 children (median age 6.5 years, range $3.65-14.5$ years) participated in this study. Children were referred to the actual MRI investigation only when they passed the training. We assessed the pass rate of the mock scanner training sessions. In addition, the quality of both structural and functional MRI (fMRI) scans was rated on a semiquantitative scale. The overall pass rate of the mock scanner training sessions was $85 / 90$. Structural scans of diagnostic quality were obtained in 81/90 children, and fMRI scans with sufficient quality for further analysis were obtained in
\end{abstract}

H. M. A. de Bie $(\varangle) \cdot$ S. Adriaanse •

H. A. Delemarre-Van de Waal

Department of Pediatrics, Pediatric Endocrinology,

VU University Medical Center,

P.O. Box 7057, 1007 MB Amsterdam, The Netherlands

e-mail: b.debie@vumc.nl

H. M. A. de Bie $\cdot$ M. Boersma $\cdot$ S. Adriaanse $\cdot$ R. J. Vermeulen

D. J. Veltman

Neuroscience Campus Amsterdam,

VU University Medical Center,

Amsterdam, The Netherlands

\section{Boersma}

Department of Clinical Neurophysiology,

VU University Medical Center,

Amsterdam, The Netherlands

\section{P. Wattjes}

Department of Radiology,

VU University Medical Center,

Amsterdam, The Netherlands
$30 / 43$ of the children. Even in children under 7 years of age, who are generally sedated, the success rate of structural scans with diagnostic quality was $53 / 60$. FMRI scans with sufficient quality were obtained in $23 / 36$ of the children in this younger age group. The association between age and proportion of children with fMRI scans of sufficient quality was not statistically significant. We conclude that a mock MRI scanner training protocol can be useful to prepare children for a diagnostic MRI scan. It may reduce the need for sedation in young children undergoing MRI. Our protocol is also effective in preparing young children to participate in fMRI investigations.

Keywords MRI · Functional MRI · Mock scanner · Training protocol

\section{R. J. Vermeulen}

Department of Child Neurology,

VU University Medical Center,

Amsterdam, The Netherlands

\section{K. J. Oostrom · J. Huisman}

Department of Medical Psychology,

VU University Medical Center,

Amsterdam, The Netherlands

D. J. Veltman

Department of Psychiatry,

VU University Medical Center,

Amsterdam, The Netherlands

H. A. Delemarre-Van de Waal

Department of Pediatrics, Leiden University Medical Center,

Leiden, The Netherlands 


\section{Introduction}

Magnetic resonance imaging (MRI) is a common diagnostic imaging modality providing exquisite anatomic detail. In recent years, functional MRI (fMRI) is increasingly being used for research on brain functioning in children. The design and dimensions of most MRI systems can be intimidating (huge machine, loud noise, and narrow bore), especially for young children. Together with a lack of comprehension, this may induce anxiety and distress, resulting in poor or non-adherence and excessive movement. MRI scans and especially fMRI scans are highly sensitive to movement artifacts. Therefore, it is difficult to obtain scans of good quality in young children, and sedation or general anesthesia is used in most patients undergoing MRI investigation under 7 years of age $[4,14$, 22]. Sedation is used even at higher ages in children with mental retardation or severe behavioral abnormalities. Due to a great increase in the number of MRI investigations in the pediatric population, MRI investigations under sedation can be delayed for several months [24]. Additionally, sedation of pediatric patients has associated risks. Among those, respiratory depression and airway obstruction are the most important $[4,7,16]$. Several factors contributing to this risk are drug overdosage, drug interactions, and the lack of established procedures to provide sedation by qualified personnel [3, 4]. Under optimal conditions, serious complications are extremely rare [6]. In contrast to MRI investigations in a diagnostic setting, most ethics committees will not allow sedation to be used in research studies involving normally developing children. In addition, task-related fMRI requires an awake and cooperative participant, which precludes sedation. Several procedures for obtaining scans of acceptable quality in young children while avoiding the use of sedation have been suggested, including acquisition during sleep, explaining the procedure of the MRI investigation using photos or a video, or using a small model of the MRI scanner to familiarize the child with the layout of the unit $[11,17,18]$. In addition, extensive preparation with a guided tour of the MRI facility and employing a step-by-step approach to introduce all necessary equipment have been described [2]. Furthermore, distraction devices such as video viewing systems or relaxation audiotapes can be used during actual MRI investigation $[10,13,19,22]$. A promising alternative is the use of a mock scanner or tunnel to prepare children for
MRI investigation [9]. For fMRI, preparation of young children with a mock scanner has been referred to previously, but details regarding a protocolized training session were not provided [25]. In the present study, we evaluate the use of a mock scanner training protocol for preparation of children of 3 to 14 years of age for both structural and functional MRI.

\section{Participants and methods}

\section{Participants}

Ninety children (median age 6.5 years, range $3.7-14.5$ years) were consecutively included and prepared according to the mock scanner training protocol. The population consisted of 47 children who visited the outpatient clinic of our hospital (MRI group) and 43 children who were recruited for a controlled study on brain development, intelligence, and cognitive outcome in children born small for gestational age (fMRI group, Dutch Trial Register: NTR 865). The children in the MRI group were referred for MRI investigation by their treating physician for usual clinical purposes. These children were referred from different departments: pediatric neurology (developmental delay $n=28$, epilepsy $n=4$, and ventriculo peritoneal drain dysfunction $n=1)$, pediatric oncology $(n=6)$, pediatric orthopedics $(n=5)$, pediatric nephrology $(n=2)$, and pediatric endocrinology $(n=1)$. The lower age limit of participating in the training protocol was set around the fourth birthday. Exclusion criteria were apparent developmental disorder and severe mental retardation precluding communication and consistent cooperation. Judgement was done by the referring physician and if necessary he or she consulted one of the members of the training team. The fMRI group consisted of 31 children born small for gestational age and 12 healthy children. This study was approved by the ethics committee of the VU University Medical Center. Written informed consent was obtained from the parents or guardians of each child. The study was performed at the VU University Medical Center. Baseline characteristics of the population are listed in Table 1.

\section{Mock scanner unit}

The mock scanner is a full-scale replica of a MRI system, without magnets. It is located at the pediatric outpatient
Table 1 Characteristics of population

\begin{tabular}{llll}
\hline & MRI group & fMRI group & Total \\
\hline Number of children & 47 & 43 & 90 \\
Age median, years (range) & $6.9(3.7-14.5)$ & $5.7(4.1-7.6)$ & $6.5(3.7-14.5)$ \\
Sex, girls:boys & $20: 27$ & $21: 22$ & $41: 49$ \\
\hline
\end{tabular}


department (Fig. 1). The mock scanner is equipped with a manually operated patient table, head coil, foam cushions, headphones, and earplugs. Speakers inside the bore reproduce the sounds of various scan sequences that can be heard during actual MRI investigations.

\section{Mock scanner training protocol}

A pediatrician or experienced child-life specialist conducted the training session. The training was held at least several days to a maximum of 3 weeks before the actual MRI investigation, enabling scheduling of an extra training session if necessary. The children entered the mock scanner unit with at least one of their parents. The training session consisted of two parts. During the first part of the training session, the children were sitting next to the mock scanner. The children were verbally instructed on why they had to undergo the MRI investigation and on the importance of minimizing motion. They were told that they had to practice in the mock scanner to become familiar with the MRI environment. The various parts of the MRI unit were demonstrated, and each step of the MRI investigation was explained. Also, the various MRI sounds were played at increasing volumes for the children to become accustomed to. To familiarize the child with these sounds, the instructor associated the sounds of the various scan sequences with familiar sounds, e.g. of a train or a ship. For this part of the training, the youngest children until the age of around seven were asked to bring a teddy bear that was actually placed in the scanner during instruction. During the second part of the training session, the children were encouraged to lie down in the mock scanner, equipped with headphones, and immobilized with foam cushions. Parents maintained physical contact by touching the child's legs and were asked to verbally encourage their child as much as possible

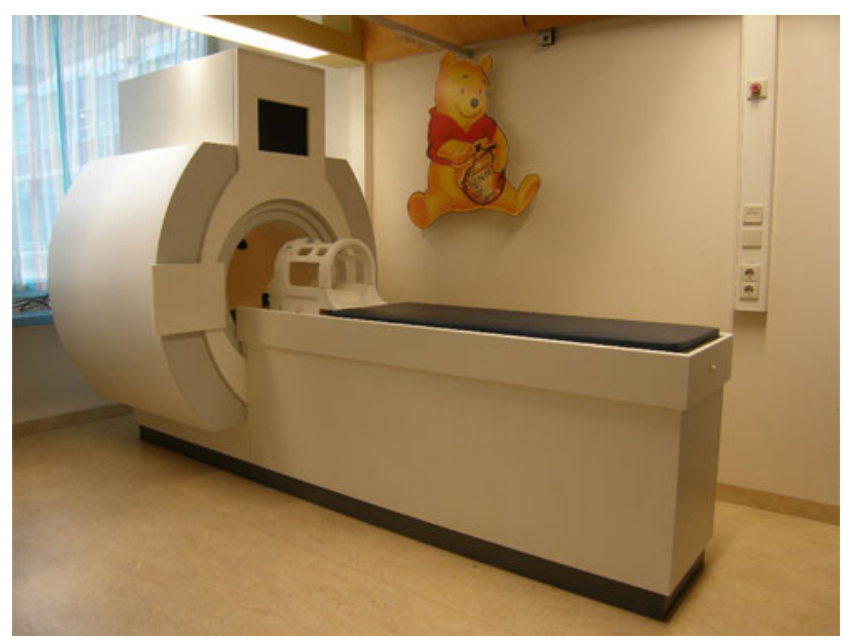

Fig. 1 Mock scanner unit at the pediatric outpatient department during the training session. We monitored motion by visual inspection of gross movements.

A training session was considered a pass when a child was able to lie still for $5 \mathrm{~min}$ in the mock scanner while the recorded sounds were heard at maximal volume. This time period was chosen because in general, single MRI runs do not last longer than approximately $5 \mathrm{~min}$. The children were given the opportunity to get an extra training session when they were able to lie still in the mock scanner, but without the recorded sounds at maximal volume, or when they had difficulties lying motionless in the scanner for $5 \mathrm{~min}$. This extra training session was planned on a separate day. A training session was recorded as failed in case of noncompletion of the training session (e.g. not able to enter the mock scanner due to excessive anxiety, inability to cooperate, and excessive movement) and if the team judged that an extra training session would not be effective. A training session lasted 30-60 min, depending on the time a child needed to get familiarized and its performance in the mock scanner.

Children were referred for the actual MRI investigation only after successful completion of the training session. Children of the MRI group who failed were referred for clinical MRI investigation with sedation. For children of the fMRI group who failed the training, the fMRI investigation was canceled. Overall pass rate of the mock scanner training protocol was defined as the proportion of children that passed the training protocol irrespective of the number of training sessions.

\section{MRI investigation and scanning protocol}

Structural images were acquired on a Siemens 1.5T MRI scanner (Siemens, Erlangen, Germany), or a GE Signa 3.0T MRI scanner (General Electric Medical Systems, Milwaukee, Wisconsin, USA). All functional images were acquired on a Siemens $1.5 \mathrm{~T}$ scanner. The MRI group underwent MRI investigation consisting of structural scan sequences only. Total MRI investigation of the MRI group varied between 20 min and $45 \mathrm{~min}$. Participants of the fMRI group had two fMRI runs and one structural reference scan. The latter study aimed to investigate declarative memory using a visual encoding task, and each fMRI run lasted 3 min $30 \mathrm{~s}$. Details regarding this study will be reported separately. Total duration of the fMRI investigation did not exceed $20 \mathrm{~min}$.

Similar to the mock scanner training session, one of the parents and the pediatrician or a child-life specialist was sitting next to the child during the actual MRI investigation, maintaining physical contact, and encouraging the child. Foam padding was used to further limit motion.

Quality rating of scans

The quality of structural MRI scans was rated according to a five-point rating scale by an experienced radiologist 
(MPW) as 1: no motion artifacts, excellent quality, 2: little motion artifacts, good quality, 3: moderate motion artifacts, acceptable quality, 4: excessive motion artifacts, poor quality, and 5: incomplete scan (Fig. 2). Scans of score 13 were considered to be of sufficient quality for diagnostic purposes. Success rate of structural scan sessions was defined as the proportion of children with structural MRI scans with score 1-3. As sedation is used frequently in children under 7 years of age, we also assessed the success rate of structural scan sessions in this younger group separately.

The quality of fMRI scans was based on head motion parameters, and was analyzed using Statistical Parametric Mapping (SPM5) software, developed by the Wellcome Department of Cognitive Neurology, London, UK (http://www.fil.ion.ucl.ac.uk). During spatial preprocessing of the data, head motion parameters were estimated. From these motion estimates, the maximum displacement in any plane across the entire run was calculated for each subject. Functional scans were rated on a three-point scale as 1: maximum displacement during fMRI run $\leq 3 \mathrm{~mm}$ (one voxel in our fMRI study design), 2: maximum displacement during fMRI runs $>3 \mathrm{~mm}$, and 3: incomplete scan. Scans with score 1 were considered to have sufficient quality for further fMRI analysis. Success rate of the fMRI scan sessions was defined as the proportion of children with fMRI scans with score 1 for both fMRI runs.

\section{Statistical methods}

Statistical analysis was performed with Statistical Package of Social Sciences (SPSS version 16.0). We used Chi squared test for trend analysis to calculate a relation between age of the child and the pass rate, or the proportion of children with good quality scans. A $p$ value of $<0.05$ was considered significant.

\section{Results}

Total group (3-14 years of age)

The overall pass rate of the mock scanner training protocol was $85 / 90$ (Table 2). Seventy-two children passed the protocolized training after one session. Nine out of 47 children from the MRI group and four out of 43 of the fMRI group needed an extra training session but could eventually be referred for actual MRI investigation. We found a positive relation between age and pass rate of the mock scanner training protocol. Older children had a higher pass rate (Chi squared test for trend $p=0.026$ ).

The overall quality of the structural MRI scans in the 45 children in the MRI group, as well as in the 40 children in the fMRI group, was high for all ages (Fig. 3). In the total sample, 81 out of 90 had scans of acceptable to excellent quality (score 1-3, Table 2). Two girls of 9 and 14 years of age completed the MRI investigation but their scans showed excessive motion artifacts. One of the girls has a general developmental disorder with a severe form of epilepsy, and the other girl has Gilles De La Tourette Syndrome. In two other children, both 4 years of age, the MRI investigation had to be aborted because of anxiety of the children. Once children had passed the mock scanner training protocol, there were no age related differences with respect to quality of the structural MRI scans.

Thirty out of 40 children who successfully completed the training protocol had fMRI scans with $\leq 3 \mathrm{~mm}$ head motion (Table 2). The association between age and proportion of

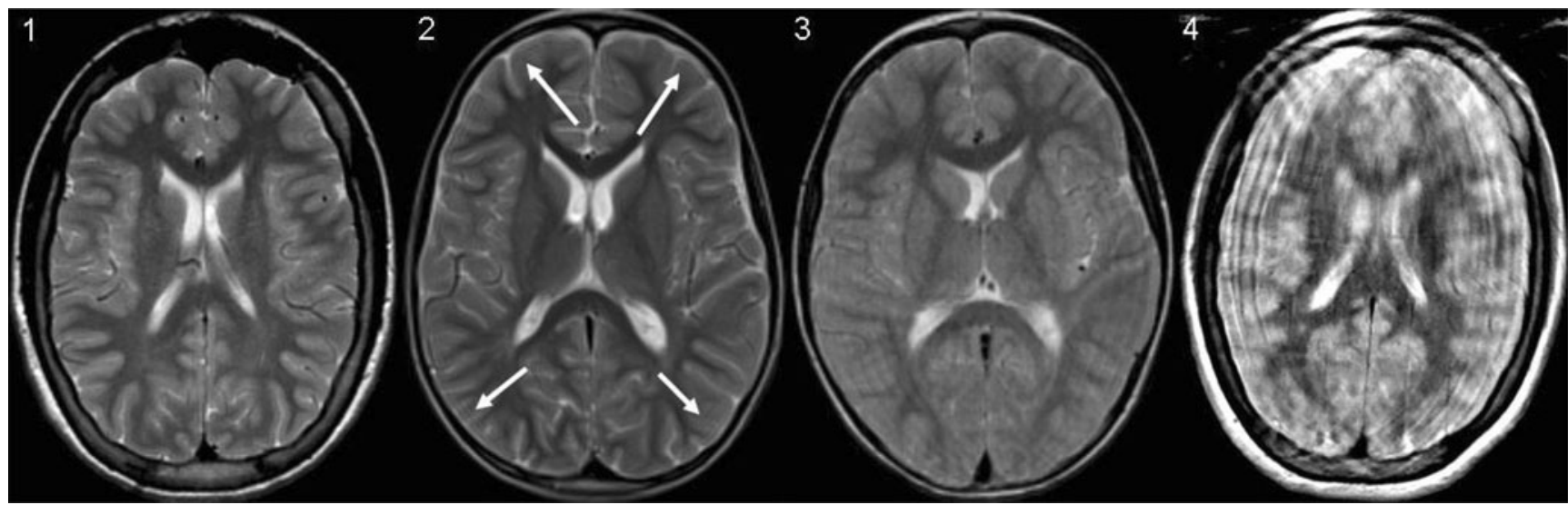

Fig. 2 Transverse sections of T2-weighted MR images through the supratentorial brain demonstrating different degrees of movement artifacts according to our rating scale. 1: no motion artifacts, excellent quality, 2: little motion artifacts (arrows), good quality, 3: moderate motion artifacts, acceptable quality, and 4: excessive motion artifacts, poor quality 
Table 2 Pass rate of mock scanner training sessions and quality of MRI investigations: total group, $n=90$

\begin{tabular}{|c|c|c|c|c|}
\hline & & $\begin{array}{l}\text { Diagnostic MRI group } \\
(n=47)\end{array}$ & $\begin{array}{l}\text { Functional MRI group } \\
(n=43)\end{array}$ & $\begin{array}{l}\text { Total } \\
(n=90)\end{array}$ \\
\hline Pass rate of mock scanner training sessions & & $45 / 47$ & $40 / 43$ & $85 / 90$ \\
\hline \multicolumn{5}{|l|}{ Quality of structural MRI scans } \\
\hline Excellent quality & Score 1 & 10 & 15 & 25 \\
\hline Good quality & Score 2 & 16 & 16 & 32 \\
\hline Acceptable quality & Score 3 & 15 & 9 & 24 \\
\hline Poor quality & Score 4 & 2 & 0 & 2 \\
\hline Incomplete MRI investigation & Score 5 & 2 & 0 & 2 \\
\hline \multicolumn{5}{|l|}{ Quality of functional MRI scans } \\
\hline Movement $\leq 3 \mathrm{~mm}$ & Score 1 & & 30 & 30 \\
\hline Movement $>3 \mathrm{~mm}$ & Score 2 & & 10 & 10 \\
\hline Incomplete MRI investigation & Score 3 & & 0 & 0 \\
\hline
\end{tabular}

children with fMRI scans of sufficient quality was not statistically significant (Fig. 4).

Children under 7 years of age

Sixty children in our population were younger than 7 years. Fifty five of them passed the mock scanner training protocol. Fifty-three of these 55 children completed the actual MRI investigation and had also structural MRI scans with score 1-3, which were thus considered useful for diagnostic purposes. As mentioned before, in two other children, both 4 years of age, the MRI investigation had to be aborted because of anxiety of the children.

When using our training protocol, 33 of 36 children under 7 years of age were able to participate in our fMRI study. Of these 33 children, 23 had fMRI scans with $\leq 3$-mm head motion and were thus useful for further analysis, so that 23/36 children from the overall group with which we started had fMRI scans of sufficient quality.

\section{Discussion}

In the present study, we demonstrate that a training protocol with a mock MRI scanner results in a high proportion of good quality structural MRI scans and also in a high proportion of good quality fMRI scans in children as young as 3 years. Until 6-7 years of age, sedation is a common practice for MRI investigations in a diagnostic setting $[14,20]$. Recently, several consensus statements and
Fig. 3 Quality of structural MRI scans grouped by age in 90 children

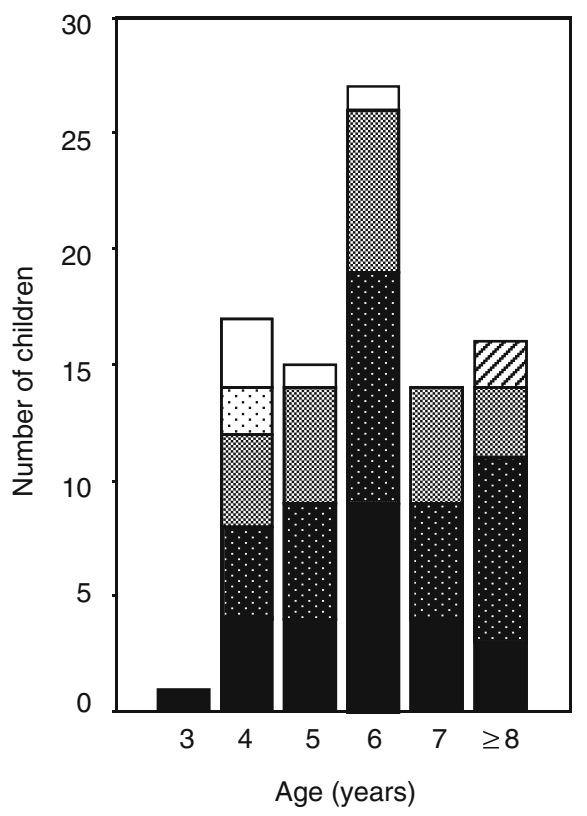


Fig. 4 Quality of functional MRI scans grouped by age in 43 children

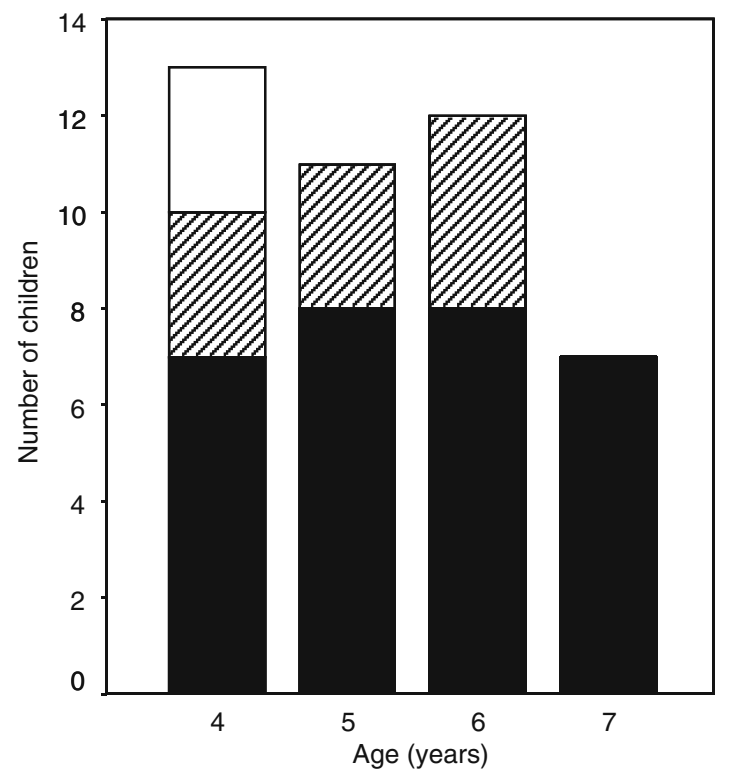

Failed training session

C Head motion $>3 \mathrm{~mm}$

Head motion $\leq 3 \mathrm{~mm}$ guidelines have been issued to achieve maximal efficiency and safety of sedation procedures [5, 21]. A potential drawback of sedation is the occurrence of serious side effects, although the overall risk is low in settings with organized sedation services $[1,7,16]$. In studies where MRI is used as a research tool, most ethics committees will not allow sedation in normally developing children. Research studies often opt for scanning during natural sleep. However, it can be very time consuming to wait for a child to fall asleep [11].

This study is strictly observational. This protocol was developed for functional imaging studies and was subsequently introduced in the outpatient clinic of our hospital. Our data show that this approach is promising. It would be very interesting to perform further controlled studies investigating specific aspects of mock scanner training sessions (e.g. the influence of noise and the exterior of the scanner).

The rate of good quality structural MRI scans of children in the age range of 4 to 7 years was $81 / 90$ and identical to results from the group of Hallowel et al. using a similar mock scanner training protocol, which was, however, for structural MRI investigations only [9]. The use of distraction devices, video viewing systems, or relaxation audiotapes have reported success rates of up to $70 \%[10,13,22]$. Scanning during sleep resulted in a high success percentage of $93 \%$ in young children 2.5-4.5 years old [17]. In older children from 5 to 17 years old success rates of $59 \%$ are reported [11]. Preparation using play therapy and a model of an MRI scanner also resulted in reduced need for sedation, but detailed information regarding success rates is lacking [18].

The training protocol enabled us to perform fMRI studies in young children. Even in children younger than 7 years we obtained a success rate of 30/43. Due to differences with regard to study populations, it is difficult to compare our success rate with other studies. The success rates using the present mock scanner protocol exceed the results of a study in which a preparation protocol including a videotape and a guided tour through the scanning laboratory was used. This protocol resulted in a success rate for completion of at least one single fMRI run of $47 \%$ in 36 healthy children between 4 and 6 years old, increasing to almost $100 \%$ in children entering their second decade [2]. A recent study using a mock scanner or tunnel to train children yielded a success rate for completion of at least one single fMRI run of $82 \%(41 / 48)$ in a mixed group of 4-6 year-old children, consisting of both healthy children and children with epilepsy [25]. Whereas this rate is even higher than in our study, it should be noted that in this study success rate was defined as the completion of one single fMRI run, in contrast to our study in which success rate was defined as the completion of the entire fMRI session. Perhaps, our success rates could be improved by more intensive or additional training sessions, especially in the younger children. Taken together, we may conclude that in very young children different preparation procedures can be used. The use of a mock scanner holds great promise (this study), but its effectiveness needs to be further explored with a controlled study design. For children over 6 years, differences between preparation procedures appear to be less relevant, because all protocols result in high success percentages.

We are aware that some children, even at older ages, can only have a successful MRI investigation following sedation or even general anesthesia. In our experience, for normally developing children, the lower age limit of participating in the training protocol is around the fourth birthday. In addition, it is obvious that besides a mock scanner, qualified and motivated personnel and good coaching during the training session and MRI investigation are indispensable for a good outcome. 
In the present study, we used fairly stringent criteria to classify fMRI scans as useful for further analysis, so that our results are likely to be conservative in this respect. We chose a level of maximum displacement during fMRI runs of $3 \mathrm{~mm}$ as our cut-off between "useful" and "not-useful" fMRI scans. For young children, there are no well-defined or accepted quality criteria for the amount of motion artifacts which are still acceptable $[8,15]$. The cut-off of $3 \mathrm{~mm}$ was chosen because this represents one voxel in our scanning protocol [25]. However, in fMRI studies in children, it is not uncommon to include scans with greater motion than $3 \mathrm{~mm}$ in the initial fMRI analysis based on visual inspection of the resulting statistical parametric maps [12, 15, 23, 25].

The actual pass rates of the mock scanner training protocol described in this study may have been somewhat inflated because for the diagnostic group, the referring physician did not include patients with severe mental retardation or apparent developmental disorder. Furthermore, inclusion was mainly based on age (lower limit of 4 years) and the estimated level of cooperativeness. This triage system seems to work well, based on the high success rates of both completion of the training protocol and scans with acceptable quality, but may be further improved by analysis of drop-outs.

In summary, the scarce data from the literature and from our own experience indicate that a training protocol with a mock MRI scanner can be applied in young children, in the age range of 4 to 7 years old, undergoing a diagnostic MRI scan, thereby reducing the need for sedation. In addition, for research studies, this study protocol provides a potential alternative to scanning young children during natural sleep.

Acknowledgements We thank S. Deen and L. Rem-Tuyn for their assistance in coaching of the children. We thank J.P.A. Kuijer and W. Bloem for their technical assistance. T. Schweigmann and V.M. Knaake are greatly acknowledged for their help with data acquisition. This work was financially supported by an educational grant from Pfizer bv, The Netherlands. The authors declare that they have no conflict of interest.

Open Access This article is distributed under the terms of the Creative Commons Attribution Noncommercial License which permits any noncommercial use, distribution, and reproduction in any medium, provided the original author(s) and source are credited.

\section{References}

1. Bluemke DA, Breiter SN (2000) Sedation procedures in MR imaging: safety, effectiveness, and nursing effect on examinations. Radiology 216:645-652

2. Byars AW, Holland SK, Strawsburg RH et al (2002) Practical aspects of conducting large-scale functional magnetic resonance imaging studies in children. J Child Neurol 17:885-890

3. Cote CJ, Karl HW, Notterman DA et al (2000) Adverse sedation events in pediatrics: analysis of medications used for sedation. Pediatrics 106:633-644
4. Cote CJ, Notterman DA, Karl HW et al (2000) Adverse sedation events in pediatrics: a critical incident analysis of contributing factors. Pediatrics 105:805-814

5. Cote CJ, Wilson S (2006) Guidelines for monitoring and management of pediatric patients during and after sedation for diagnostic and therapeutic procedures: an update. Pediatrics 118:2587-2602

6. Cravero JP, Beach ML, Blike GT et al (2009) The incidence and nature of adverse events during pediatric sedation/anesthesia with propofol for procedures outside the operating room: a report from the Pediatric Sedation Research Consortium. Anesth Analg 108:795-804

7. Cravero JP, Blike GT, Beach M et al (2006) Incidence and nature of adverse events during pediatric sedation/anesthesia for procedures outside the operating room: report from the Pediatric Sedation Research Consortium. Pediatrics 118:1087-1096

8. Epstein JN, Casey BJ, Tonev ST et al (2007) Assessment and prevention of head motion during imaging of patients with attention deficit hyperactivity disorder. Psychiatry Res 155:75-82

9. Hallowell LM, Stewart SE, de Amorim ESCT, Ditchfield MR (2008) Reviewing the process of preparing children for MRI. Pediatr Radiol 38:271-279

10. Harned RK, Strain JD (2001) MRI-compatible audio/visual system: impact on pediatric sedation. Pediatr Radiol 31:247-250

11. Johnson K, Page A, Williams H et al (2002) The use of melatonin as an alternative to sedation in uncooperative children undergoing an MRI examination. Clin Radiol 57:502-506

12. Klaver P, Lichtensteiger J, Bucher K et al (2008) Dorsal stream development in motion and structure-from-motion perception. Neuroimage 39:1815-1823

13. Koch BL (2008) Avoiding sedation in pediatric radiology. Pediatr Radiol 38(Suppl 2):S225-S226

14. Lawson GR (2000) Controversy: sedation of children for magnetic resonance imaging. Arch Dis Child 82:150-153

15. Lichtensteiger J, Loenneker T, Bucher K et al (2008) Role of dorsal and ventral stream development in biological motion perception. Neuroreport 19:1763-1767

16. Malviya S, Voepel-Lewis T, Tait AR (1997) Adverse events and risk factors associated with the sedation of children by nonanesthesiologists. Anesth Analg 85:1207-1213

17. Nordahl CW, Simon TJ, Zierhut C et al (2008) Brief report: methods for acquiring structural MRI data in very young children with autism without the use of sedation. J Autism Dev Disord 38:1581-1590

18. Pressdee D, May L, Eastman E, Grier D (1997) The use of play therapy in the preparation of children undergoing MR imaging. Clin Radiol 52:945-947

19. Raschle NM, Lee M, Buechler R et al. (2009) Making MR imaging child's play_ pediatric neuroimaging protocol, guidelines and procedure. J Vis Exp

20. Rosenberg DR, Sweeney JA, Gillen JS et al (1997) Magnetic resonance imaging of children without sedation: preparation with simulation. J Am Acad Child Adolesc Psychiatry 36:853-859

21. Scotttish Intercollegial Guidelines network: safe sedation of children undergoing diagnostic and therapeutic procedures. A national guideline.;Edinburgh: Royal College of Physicians. 2002

22. Smart G (1997) Helping children relax during magnetic resonance imaging. MCN Am J Matern Child Nurs 22:236-241

23. Supekar K, Musen M, Menon V (2009) Development of large-scale functional brain networks in children. PLoS Biol 7:e1000157

24. Wachtel RE, Dexter F, Dow AJ (2009) Growth rates in pediatric diagnostic imaging and sedation. Anesth Analg 108:1616-1621

25. Yerys BE, Jankowski KF, Shook D et al (2009) The fMRI success rate of children and adolescents: typical development, epilepsy, attention deficit/hyperactivity disorder, and autism spectrum disorders. Hum Brain Mapp 30:3426-3435 